\title{
Patient safety \\ Rapid response systems
}

Kenneth M Hillman

MD, FRCA, FCICM

Professor of Intensive Care

Jack Chen

MBBS, PhD, MBA

Associate Professor of Health Services Research

Daryl Jones

$M D, F R A C P, F C I C M$,

Associate Professor of

Intensive Care ${ }^{2}$

1 University of

New South Wales,

Sydney, NSW.

2 Austin Health,

Melbourne, VIC.

k.hillman@unsw.edu.au

doi: $10.5694 / \mathrm{mjal} 4.01088$ apid response systems (RRSs) have become a routine part of the way patients are managed in general wards of acute care hospitals (Box 1). ${ }^{1}$ They are used in most hospitals in Australasia, North America and the United Kingdom and are increasingly being used in other parts of the world. They operate across the whole hospital and aim for early identification of seriously ill patients, at-risk patients and patients whose condition is deteriorating, using abnormal observations and vital signs (calling criteria). If any of these criteria are breached, the bedside nurse or doctor triggers a rapid response by clinicians who have the expert skills, knowledge and experience to initiate a coordinated response to any hospital medical emergency.

Traditionally, the most junior doctor and the bedside nurse were the first-line management team caring for patients in acute care hospitals. Interns were expected to assess and manage patients with deteriorating conditions, with little experience in the complexities involved in caring for more seriously ill patients. In the early 1990s, it started to become evident that many potentially preventable deaths and serious adverse events were occurring in acute care hospitals. ${ }^{2,3}$

Errors were ascribed to the system in which clinicians operated, rather than to individual incompetence. ${ }^{4}$ Hospitals operated in silos, where patients were admitted under a specialist in one area of medicine. This has certain strengths, such as the admitting doctor being ultimately responsible for the patient's care. It had been a successful mode of operation for some time, but several changes have made it less effective. ${ }^{5}$ The population of patients in hospitals has shifted from relatively young patients with a single diagnosis to increasingly older patients with multiple comorbidities who undergo more complicated diagnostic procedures and treatment regimens. ${ }^{6}$ The needs of these older patients cannot necessarily be met by a specialist with limited experience outside his or her own area of expertise. ${ }^{7}$ The consultant, even if immediately available, may not have the appropriate skills to recognise and manage seriously ill patients requiring critical care interventions. Similarly, the consultant's team of trainees, although more immediately available, may not have had the training necessary to manage seriously ill patients.

Patients with deteriorating conditions were not being recognised. More than $80 \%$ of those who suffered a cardiac arrest in hospital had documented deterioration in vital signs in the 8 hours before the arrest. ${ }^{8}$ More than $50 \%$ of those who died in hospital without a do-not-resuscitate order had severe antecedent derangements in vital signs. ${ }^{9}$ About $70 \%$ of patients admitted to an intensive care unit (ICU) from the general wards had vital sign abnormalities

\section{Summary}

Rapid response systems (RRSs) are one of the first organisation-wide, patient-focused systems to be developed to prevent potentially avoidable deaths and serious adverse events such as cardiac arrests.

RRSs identify seriously ill and at-risk patients and those whose condition is deteriorating, using abnormal vital signs and observations that trigger an urgent response by staff who are able to deal with any medical emergency.

RRS teams also respond to staff concern - any bedside nurse or doctor who is concerned about his or her patient can seek assistance.

RRSs require the support of the whole hospital. This includes resources, educational programs and agreed ways of evaluating RRS effectiveness.

RRSs may reduce deaths by up to one-third and cardiac arrests by up to $50 \%$.

for at least 8 hours before being admitted to the ICU. ${ }^{10}$ Patients with deteriorating conditions were also managed suboptimally. A recent report from the United Kingdom found that the three most common reasons for potentially avoidable mortality in UK hospitals were mismanagement of deterioration (35\%), failure of prevention (26\%) and deficient checking and oversight $(10 \%) .{ }^{11}$ RRSs have the potential to overcome all these problems.

\section{Establishing a system around patient needs}

It is common in hospitals for clinicians in one specialty to seek the opinion of clinicians in another specialty by requesting a consultation. Typically, this is not time critical. However, when a patient's condition is deteriorating, the consultation must be as prompt as possible. For this to occur, an agreed way of defining at-risk patients is needed. This underpins the need for a standardised and objective set of calling criteria superseding the usual consultation process. Early intervention is more effective than waiting until a patient is so seriously ill that he or she requires expensive and invasive management in an ICU or, even worse, waiting until he or she suffers a preventable cardiac arrest or dies a preventable death. ${ }^{1}$

\section{Vital signs}

Before the widespread implementation of RRSs, there was little research into one of the most common interventions in acute care hospitals - the measurement of vital signs. Vital signs have been routinely measured and charted since Florence Nightingale used them for hospitalised patients in the Crimean War. The largest study 
1 Features of a rapid response system

Defines seriously ill patients, at-risk patients and patients whose condition is deteriorating using abnormal observations and vital signs (calling criteria)

- Provides rapid response to seriously ill patients and those whose condition is deteriorating

Operates across the whole organisation

Is designed around patient needs

De-emphasises the usual hierarchies and interprofessional barriers

Provides rapid consultation by experts in critical illness

on RRS effectiveness found that almost $50 \%$ of patients who died, had a cardiac arrest or were admitted to an ICU did not have vital signs measured before the event. ${ }^{12}$ Respiratory rate, the most accurate predictor of serious illness, is often not measured and, when it is measured, the measurement is often inaccurate. ${ }^{12,13}$ These findings have focused attention on the appropriate frequency for vital sign measurement, especially because hospitalised patients in general wards are at high risk of clinical deterioration. ${ }^{11}$ Deterioration in a patient's condition can conceivably occur in the period during which vital signs are not usually measured.

\section{Calling criteria}

Vital sign abnormalities include: low systolic blood pressure (usually $<90 \mathrm{mmHg}$ ); high or low respiratory rate (eg, $<4$ breaths/min or $>30$ breaths/min); and abnormal pulse rate (eg, $<40$ beats $/ \mathrm{min}$ or $>140$ beats $/ \mathrm{min}$ ). Potentially life-threatening observational abnormalities include seizures, airway obstruction and sudden decrease in level of consciousness. Staff concern is also an important criterion, empowering bedside nurses or doctors to seek timely assistance if they are worried about a patient who does not fit any other criterion. In mature systems, staff concern is a common reason for urgent assistance. ${ }^{14}$ Oxygen saturation abnormality, when available, is also a useful criterion.

Australian hospitals usually employ an RRS in which one calling criterion triggers a response. Hospitals in other countries may use scores, by adding vital sign abnormalities to trigger different levels of response. ${ }^{15}$ This could add a level of complexity and inaccuracy, and might encourage clinicians to focus on numbers rather than observation of the patient. It also excludes staff concern as a reason for seeking urgent attention.

Some centres are exploring the concept of encouraging family and visitors to trigger an urgent response. It is early days but, so far, there does not appear to be misuse of the system. ${ }^{16}$ Use of pathology results to identify patients at an even earlier stage in illness has also been explored. ${ }^{17}$ Although objective calling criteria are important, awareness of the RRS in itself can change an organisation's culture, moving it from a traditional hierarchical and silo-based one to one with universal awareness that there are at-risk patients in a hospital and timely assistance is available.

\section{The response}

As with calling criteria, there is much variation in how organisations provide an urgent response. Some hospitals maintain separate cardiac arrest and rapid response teams. In the UK, it is common to have an outreach system, indicators where nurses pre-emptively identify and manage at-risk patients across the hospital. ${ }^{18} \mathrm{~A}$ two-tiered system, where a member of the admitting team may be called for less serious abnormalities, is used in many organisations. ${ }^{19}$

Based on the evidence that hospitals suboptimally recognise and manage seriously ill patients, ${ }^{1-4,8-10}$ it is important to involve clinicians who have the appropriate training when caring for these patients, who often have complex needs. It is not surprising, therefore, that many rapid response teams use ICU staff. ${ }^{20}$ However, depending on the hospital setting, the urgent response could be provided by a doctor, nurse or paramedic, or by staff from any department in the hospital, as long as they have the appropriate skills, knowledge and experience. ${ }^{21}$

\section{Other factors}

Implementing an organisation-wide system such as an RRS involves challenging the way clinicians interact, bypassing entrenched hierarchies and constructing a system centred on patient needs. This requires more than standardised calling criteria and a rapid response (Box 2). All clinicians in the hospital must be aware of the system and support it. Similarly, senior administrators need to endorse and resource the system. An organisation-wide education program is required to teach staff how the system works and to empower people to call for assistance when needed.

It is also important to continually monitor the system and close the loop by making outcome indicators available to people at all levels of the organisation, especially to those responsible for and participating in the system. ${ }^{22}$ Some outcome indicators include cardiac arrest rates (which usually range between 0.5 and 6.0 cardiac arrests per 1000 admissions) and crude mortality rates. To make mortality rates more meaningful, patients with do-notresuscitate orders are excluded. Data on cardiac arrests and deaths can be further refined by examining whether there were calling criteria that were not responded to appropriately in the 24 hours before the event. This gives insight into potential preventability. Delays in the rapid response can also be a useful indicator of the system's effectiveness. ${ }^{21}$ Another important outcome indicator is the number of calls per 1000 admissions — an increase in the rate of calls is associated with reductions in mortality and cardiac arrest rates. ${ }^{23}$

\section{Do rapid response systems work?}

ICUs and RRSs are both systems for managing seriously ill and at-risk patients, but little robust research has been done to show the effectiveness of either. The general

\section{Strategies for maximising the impact of a hospital rapid response system}

Engage the support of all doctors and nurses

Ensure that there is leadership and support from senior hospital executives

Implement strategies that promote hospital-wide awareness of the system

Ensure an urgent response to any staff concern, whether life-threatening or not

Ensure a 24/7 response by staff with appropriate skills, knowledge and experience

Build outcome indicators into the system and ensure targeted feedback of data

Conduct regular multidisciplinary meetings to discuss individual cases and outcome 
intuitive principle with such systems is matching the right people - with the right skills and knowledge with the right patients at the right time.

It has been established that ICUs and RRSs identify and treat patients with a similar level of mortality risk. ${ }^{24}$ In other words, the boundaries between patients in general wards and patients in ICUs and high-dependency units (HDUs) are becoming blurred. One of the functions of an RRS is to act as a triage system, to identify sick ward patients who require ICU or HDU therapy. A 200-bed hospital with a 20-bed ICU will probably have less need for an RRS than an 800-bed hospital with a 10-bed ICU, as the more at-risk patients will already be in a highly monitored environment. In each hospital, RRS use also depends on patient casemix, average level of comorbidity and types of interventions undertaken.

Nevertheless, RRSs have been subject to evaluation. Not surprisingly, single-centre, before-and-after studies have almost universally shown significant reductions in outcome indicators such as mortality and cardiac arrest rates. ${ }^{25,26}$ Many of these studies were conducted by one or two "champions" who provided clinical leadership. The largest cluster randomised trial was underpowered and produced inconclusive results, ${ }_{1}^{12}$ possibly due to the contamination of control hospitals, less than satisfactory implementation and adherence, and variability in the effectiveness of implementation. Nevertheless, in a post-hoc analysis, the study did show a significant reduction in mortality in adult intervention hospitals. ${ }^{23}$ The largest meta-analysis on RRSs has shown $21 \%$ and $38 \%$ reductions in mortality and cardiac arrest rates, respectively, in paediatric hospitals, and a $34 \%$ reduction in cardiac arrest rates in adult hospitals. ${ }^{20}$ However, it is impossible to randomly assign patients to a group that receives early intervention by a rapid response team and a group that does not. Similarly, because of the almost universal uptake of RRSs in many countries, ${ }^{1}$ it is difficult to randomly assign hospitals. Other research methods must, therefore, be used. A recent study has shown a strong correlation between uptake of RRSs in New South Wales hospitals and reductions in cardiac arrest and cardiac arrest-related mortality rates, both of which decreased by about $50 \%$ over an 8 -year period. ${ }^{27}$

Research now needs to shift to determining the most effective response teams, evaluating the sensitivity and specificity of calling criteria, assessing the cost-effectiveness of implementing RRSs, and defining the most effective RRS implementation strategies. Moreover, possible negative effects of RRS implementation - such as de-skilling of staff and putting excessive pressure on existing resources - need to be evaluated.

In the coming months, the Journal will publish a series of articles that explore how RRSs have changed our approach to patient safety, how RRSs have influenced end-of-life care in acute care hospitals, and how the use of cardiopulmonary resuscitation and cardiac arrest teams is changing.

Acknowledgements: Kenneth Hillman and Jack Chen are supported by National Health and Medical Research Council Project Grants (APP1009916 and APP1020660).
Competing interests: No relevant disclosures.

Provenance: Commissioned; externally peer reviewed.

1 Jones DA, DeVita MA, Bellomo R. Rapid response teams. N Engl J Med 2011; 365: 139-146.

2 Brennan TA, Leape LL, Laird NM, et al. Incidence of adverse events and negligence in hospitalized patients - results from the Harvard Medical Practice Study I. NEngl J Med 1991; 324: 370-376.

3 Wilson RM, Runciman WB, Gibberd RW, et al. The Quality in Australian Health Care Study. Med J Aust 1995; 163: 458-471.

4 Kohn LT, Corrigan JM, Donaldson MS, editors. To err is human: building a safer health system. Washington, DC: National Academy Press, 2000.

5 Hillman K. The changing role of acute care hospitals. Med J Aust 1999; 170 : 325-328.

6 Adhikari NKJ, Fowler RA, Bhagwanjee S, Rubenfeld GD. Critical care and the global burden of critical illness in adults. Lancet 2010; 375: 1339-1346.

7 Donni-Lenhoff FG, Hedrick HL. Growth of specialization in graduate medical education. JAMA 2000; 284: 1284-1289.

8 Schein RM, Hazday N, Pena M, et al. Clinical antecedents to in-hospital cardiopulmonary arrest. Chest 1990; 98: 1388-1392.

9 Hillman KM, Bristow PJ, Chey T, et al. Antecedents to hospital deaths. Intern Med J 2001; 31: 343-348.

10 Hillman KM, Bristow PJ, Chey T, et al. Duration of life-threatening antecedents prior to intensive care admission. Intensive Care Med 2002; 28: 1629-1634.

11 Donaldson LJ, Panesar SS, Darzi A. Patient-safety-related hospital deaths in England: thematic analysis of incidents reported to a national database, 2010-2012. PLoS Med 2014; 11: el001667.

12 Hillman K, Chen J, Cretikos M, et al; MERIT Study Investigators. Introduction of the medical emergency team (MET) system: a clusterrandomised controlled trial. Lancet 2005; 365: 2091-2097.

13 Cretikos MA, Bellomo R, Hillman K, et al. Respiratory rate: the neglected vital sign. Med J Aust 2008; 188: 657-659.

14 Chen J, Bellomo R, Hillman K, et al; MERIT Study Investigators for the Simpson Centre and the ANZICS Clinical Trials Group. Triggers for emergency team activation: a multicenter assessment. J Crit Care 2010; 25: 359.el-369.el.

15 Chen J, Ou L, Hollis SJ. How accurate are the different predictive models in identifying deteriorating patients? The ViEWS may not be as clear as we first thought. Crit Care Med 2014; 42: 986-987.

16 Gerdik C, Vallish RO, Miles K, et al. Successful implementation of a family and patient activated rapid response team in an adult level 1 trauma center. Resuscitation 2010; 81: 1676-1681.

17 Loekito E, Bailey J, Bellomo R, et al. Common laboratory tests predict imminent death in ward patients. Resuscitation 2013; 84: 280-285.

18 Esmonde L, McDonnell, Ball C, et al. Investigating the effectiveness of critical care outreach services: a systematic review. Intensive Care Med 2006; 32: 1713-1721.

19 Clinical Excellence Commission. Between the Flags project: the way forward. Sydney: CEC, 2008. http://www.cec.health.nsw.gov. au/_documents/programs/between-the-flags/2013/btf-the-wayforward-2008.pdf (accessed Jul 2014).

20 Chan PS, Jain R, Nallmothu BK, et al. Rapid response teams. A systematic review and meta-analysis. Arch Intern Med 2010; 170: 18-26.

21 Hillman KM. Run, don't walk. Crit Care Med 2012; 40: 2712-2713.

22 Hillman K, Alexandrou E, Flabouris M, et al. Clinical outcome indicators in acute hospital medicine. Clin Intensive Care 2000; 11: 89-94.

23 Chen J, Bellomo R, Flabouris A, et al; MERIT Study Investigators for the Simpson Centre and the ANZICS Clinical Trials Group. The relationship between early emergency team calls and serious adverse events. Crit Care Med 2009; 37: 148-153.

24 Buist M, Bernard S, Nguyen TV, et al. Association between clinically abnormal observations and subsequent in-hospital mortality: a prospective study. Resuscitation 2004; 62: 137-141.

25 Bellomo R, Goldsmith D, Uchino S, et al. A prospective before-and-after trial of a medical emergency team. Med J Aust 2003; 179: 283-287.

26 Sharek PJ, Parast LM, Leong K, et al. Effect of a rapid response team on hospital-wide mortality and code rates outside the ICU in a children's hospital. JAMA 2007; 298: 2267-2274.

27 Chen J, Ou L, Hillman K, et al. The trends of cardiopulmonary arrest and mortality and their association with rapid response system expansion. Med J Aust 2014; 201: 167-170. 